\title{
CASUÍSTICA DE PRIMATAS DO LABORATÓRIO DE MEDICINA ZOOLÓGICA DO HOSPITAL VETERINÁRIO DA UNIVERSIDADE FEDERAL DO PARANÁ DE 2017 A 2020
}

\author{
(Case series of primates from the Zoological Medicine Laboratory of Paraná Federal University Veterinary \\ Hospital from 2017 to 2020
}

Fernanda Taques Wendt, Vivian Ferreira Rech, Mariana Reffatti de Oliveira, Thaiza Regina de Lima, Rafaella Martini, André Saldanha, Nagaissa Daniele Reinhardt, Rogerio Ribas Lange

Universidade Federal do Paraná, Curitiba, Paraná, Brasil.

*Correspondência: fefawendt@ufpr.br

RESUMO: A infraordem Platyrrhini, representada pelos primatas do Novo Mundo, é composta por 18 gêneros em cinco famílias, que ocorrem em ampla distribuição geográfica no Brasil e atuam como indicadores de equilíbrio ecológico. O impacto antrópico em seus habitats afeta suas populações e, enquanto algumas espécies se adaptam às mudanças, outras entram em declínio. Foi realizado um levantamento da casuística de primatas encaminhados pelo Instituto Água e Terra do Paraná (IAT) ao Laboratório de Medicina Zoológica do Hospital Veterinário da Universidade Federal do Paraná (UFPR) em Curitiba, entre os anos de 2017 e 2020. Nesse período houve atendimentos de primatas de outras origens, que foram desconsiderados para o estudo. Foram recebidos 30 primatas de quatro espécies, sendo 14 (36\%) saguis-de-tufo-preto (Callithrix penicillata), oito (26\%) bugios-ruivos (Alouatta guariba), sete (23\%) saguis-de-tufo-branco (Callithrix jacchus), e um (3\%) macaco-prego (Sapajus apela). A maioria (56\%) era do sexo feminino. Dezessete animais (56\%) foram submetidos a procedimentos cirúrgicos, sendo nove ováriohisterectomias (52\%), cinco orquiectomias (29\%) e três procedimentos odontológicos (17\%). Em 12 animais $(40 \%)$, foi constatado traumatismo, cinco apresentando trauma crânio encefálico. As castrações eram eletivas e foram realizadas apenas em saguis, a pedido do IAT. Os saguis são primatas nativos do nordeste e cerrado brasileiro, e foram introduzidos no sul e sudeste do país, onde ocupam áreas de fragmentos florestais, praças e parques. As espécies introduzidas representam grande ameaça para a diversidade biológica nativa, e a esterilização de animais hígidos é uma estratégia de manejo que tem como objetivo amenizar os impactos causados pela espécie, reduzindo a sua proliferação (SILVA, SILVA e TERRA, 2018). Os dados obtidos refletem a capacidade de expansão da espécie invasora e a necessidade de programas de controle na região. Alguns primatas vêm migrando para os centros urbanos, onde estão mais predispostos a sofrer traumatismos por ataque de animais domésticos, eletrocussão, atropelamentos, ou são vítimas da violência humana devido ao receio equivocado de transmissão de febre amarela. Por estes motivos, a segunda maior causa de atendimentos em primatas foi traumatismo, como o crânio encefálico. Em estudo de Maders (2016) sobre a casuística de primatas recebidos no Hospital Veterinário da Universidade Federal do Rio Grande do Sul, a principal causa de atendimentos em primatas $(21,56 \%)$ era relacionada a casos de trauma, entretanto não executaram esterilizações em saguis de vida livre. O fato de a maior casuística de primatas encaminhados ao Hospital Veterinário UFPR estar relacionada a procedimentos de controle de natalidade de espécies invasoras e suporte a animais traumatizados, reforça a importância da adoção de medidas de proteção para conservação das espécies de primatas nativas, aliados à conscientização da população.

Palavras-chave: espécies exóticas; medicina zoológica; saguis; trauma

\section{Referências}

MADERS, P. R. Estudo retrospectivo dos casos de primatas neotropicais atendidos no Preservas - UFRGS. 2016. 27 f. Tese (Graduação em Medicina Veterinária) - Faculdade de Veterinária, Universidade Federal do Rio Grande do Sul, Porto Alegre.

SILVA, D. F., SILVA, E. B., TERRA, A. P. Controle Populacional de espécies invasoras por meio de laqueadura e vasectomia em primatas Callithrix penicillata. relato de caso. Veterinária e Zootecnia, São Paulo, v. 25, n. 1, 2018. 